\title{
Research on a rotational speed control strategy of the mandrel in a rotary steering system
}

https://doi.org/10.1515/phys-2018-0085

Received July 30, 2018; accepted August 20, 2018

\begin{abstract}
Rotary steering systems are cutting-edge, intelligent oil drilling and steering equipment developed at the end of the twentieth century. The core function of the bit pointing rotary steering system is to control the eccentric shaft with bit pointing function to track the outer drill collar for reverse rotation [1]. When the relative speed thereof is offset, the mandrel tool face remains stationary, and the directive function of the drill bit is realized [2]. Therefore, it is important to precisely control mandrel rotational speed and make it follow the outer drill collar to conduct faceto-face rotation at all times. A double closed loop control method for realizing rotational speed loop and position loop through controlling mandrel rotational speed is proposed in this paper. Relative rotational speed of drill bit shaft (or mandrel) and drill collar of zero can be realized within the shortest time, thereby realizing rapid and precise rotational speed tracking. Relative positions of mandrel and drill collar at several angles on $360^{\circ}$ circumferences, thereby proving the feasibility of the method. The method can provide a technical reference and basis for prototype production of directional rotary steering system [3].
\end{abstract}

Keywords: rotary steering, directional, rotational speed control, rotational speed tracking

PACS: 02.30.Yy, 02.60.Ed

\footnotetext{
Liancheng Zhang: Liancheng Zhang: School of Geophysics and Information Technology, China University of Geosciences, Beijing, China; CNPC Drilling Research Institute, Beijing, China, E-mail: zhlc_dri@cnpc.com.cn

^Corresponding Author: Guibin Zhang: School of Geophysics and Information Technology, China University of Geosciences, Beijing, China, E-mail: gbzhang@cugb.edu.cn

John Spillian: Texas A\&M University at Commerce, Texas, 75428, USA, E-mail: John.Spillian@outlook.com
}

\section{Introduction}

Rotary steering technology includes electronic measurement technology; power electronics technology; and multidisciplinary, comprehensive and modern frontier oil drilling technology. Rotary steering technology can adjust the mandrel direction in real time during drilling, and the mandrel drills smoothly according to the set drilling trajectory. Therefore, rotary steering technology not only can simplify the procedure of drilling operation, save drilling time, improve the drilling operation accuracy and reduce drilling cost, but also can achieve better hole cleaning effect, higher orbit control precision and stronger displacement extension ability compared with the traditional guide drilling tools. It has an excellent application in complex oil and gas reservoir ultra-deep well, difficult directional well, cluster well, large displacement horizontal well and other special craft wells. It can greatly improve operation efficiency and speed, reduce operation cost, and reduce drilling safety accidents at the same time [3, 4].

The currently used rotary steering wheel technology mainly includes pushing, directional and compound modes. The directional steering wheel system does not depend on the well wall stratigraphic conditions compared with other rotary steering systems; the deflection ability is also stronger and the technology can better adapt to the complex underground working environment [5].

Bit pointing rotary steering tools drive mandrel rotation through the motor in the drill collar. The mandrel and the drill collar are rotated in opposite directions. When the mandrel rotational speed and the drill collar rotational speed are equal, the drill bit is pointing to a set angle, thereby realizing guiding operation. The rotational speed of the mandrel must be adjusted in real time in order to realize the directing operation, thereby it can track the drilling speed of the drill collar, thereby maintaining the speed thereof equal with opposite directions. The mandrel drive and speed regulation are the most critical parts of the rotary steering system. A rotational speed control method of directional rotary steering system mandrel is proposed in the paper [6]. In this paper, a directional rotary steering system mandrel rotational speed control method is pro- 
posed. Double closed-loop control of speed loop and tool face angle position loop can be realized through controlling mandrel rotational speed, thereby realizing rapid, stable and accurate steering function.

\section{Control strategy}

Any particle on the rotating mandrel is observed under the rotating state of the mandrel. The linear velocity and acceleration are changed at all times, and they belong to objects of variable speed circular motion. The mandrel rotational speed is measured at any time through a rotational speed sensor, thereby creating a closed loop control system [7]. The rotational speed tracking control method proposed in Reference 7 is shown as follows: a neural network is utilized to approximate a complex nonlinear function, and adaptive control is adopted to realize nature speed tracking [8]. Linear changes are introduced in order to realize rotational speed tracking of permanent magnet synchronous motors in Reference 8, and the time-varying models are changed into linear quantitative models [9]. These methods of rotational speed tracking are commonly used rotational speed and position tracking modes at present. Its essence is shown as follows: the gap of real value and target value can be constantly reduced through scale amplifying, integral and differential operation according to a mathematical model by controlling and measuring the difference value between target rotational speed and actual rotational speed, thereby realizing rotational speed tracking, and the only difference lies in selection closed loop error [10, 11]. The directional rotary steering system controls the mandrel wheel speed. Meanwhile, the steering of the drill bit tool face angle also can be realized. If it is necessary to investigate the rotational speed difference between the mandrel and drill collar as well as position difference between the mandrel steering tool face and target tool face at the same time in accordance with the traditional PID control strategy, the directive function of rotary steering drill bit can be realized through double closed-loop control. On the one hand, the control strategy becomes complex, while on the other hand the sensibility of the directional control is reduced at the same time [12]. A rotational speed tracking control method is introduced in this paper, the double closed-loop control of rotational speed loop and tool face angle position loop can be realized through mandrel rotational speed control, thereby simplifying the control process, and improving the control sensitivity. The control method is introduced as follows through several different initial positions of the mandrel.

\subsection{Mandrel initial state analysis and control decision}

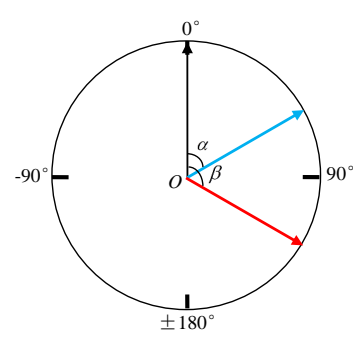

Figure 1: Tool face angle

Figure 1 shows plan sketch of tool face angle. The tool face angle circumference is divided into $-180^{\circ} \sim+180^{\circ}$, initial tool face angle is $\beta_{0}$, and target tool face angle is $\beta$. The mandrel undergoes look-ahead or lag accommodation according to actual conditions in order to ensure that $\alpha$ can reach $\beta$ along the shortest path in one circumference, and the tool face angle can be changed clockwise or counterclockwise. It is necessary to accelerate the mandrel followed by deceleration or decelerate the mandrel followed by acceleration adjustment for decision-making; firstly in order to change the tool face angle under the condition of equal mandrel rotational speed and drill collar rotational speed at the initial time. The flow chart is shown in Figure 2.

Figure 2 shows the flow chart of mandrel look-ahead or lag accommodation decision.

(1) Obtain initial tool face angle and target tool face angle from main control unit;

(2) Calculate difference value between target and initial tool face angle.

Plan A: lag accommodation is implemented if |difference value $\mid \leq 180^{\circ}$ when difference value $>0^{\circ}$, namely mandrel motor is decelerated followed by acceleration for accommodation, the tool face angle can be changed clockwise; plan B: look-ahead accommodation is implemented when difference value $\leq 0^{\circ}$, namely mandrel motor is accelerated followed by deceleration for accommodation, and tool face angle can be changed counterclockwise;

Plan B: look-ahead accommodation is implemented if |difference value $\mid>180^{\circ}$ when difference value $>0^{\circ}$, namely mandrel motor is accelerated followed by deceleration for accommodation, the tool face angle can be changed counterclockwise; plan A: lag accommodation is implemented when difference value $\leq 0^{\circ}$, namely mandrel 
motor is decelerated followed by acceleration for accommodation, and tool face angle can be changed clockwise.

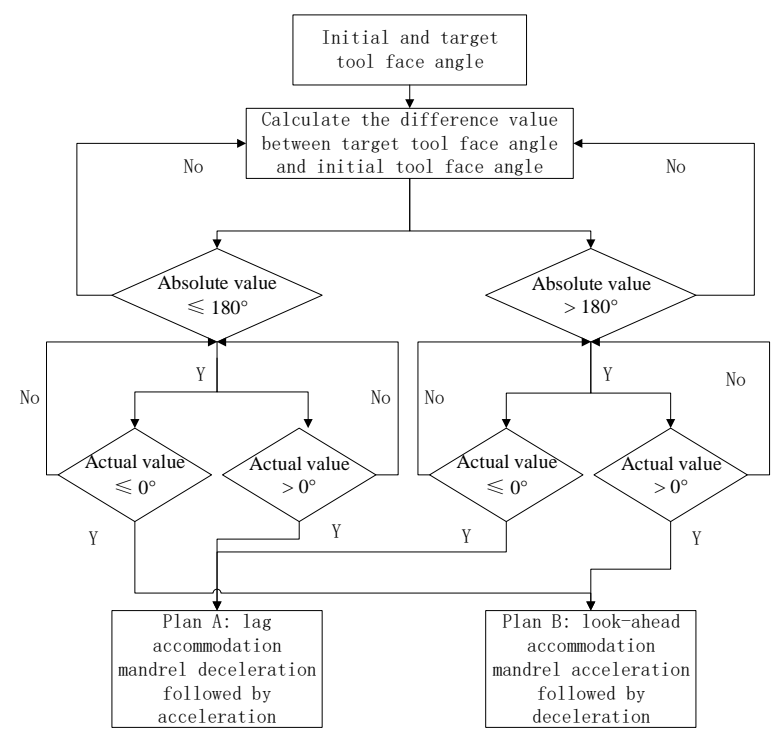

Figure 2: Mandrel look-ahead or lag accommodation decision

\subsection{Mandrel rotational speed control algorithm}

Figure 3 shows the control algorithm flow chart of mandrel look-ahead or lag speed control.

Mandrel motor rotational speed at one time point is shown as follows:

$$
n(i)=n(i-1)+\Delta n(i)
$$

Plan A lag accommodation - mandrel motor deceleration followed by acceleration is adopted as an example. Mandrel rotational speed changes are divided into three stages for control:

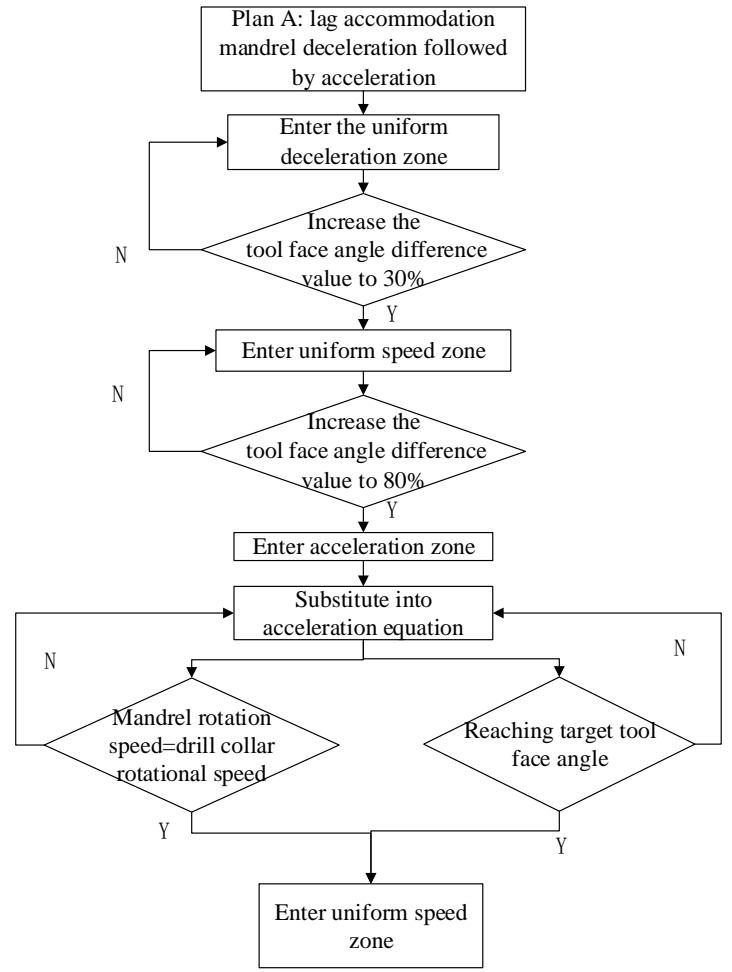

(a) Plan A

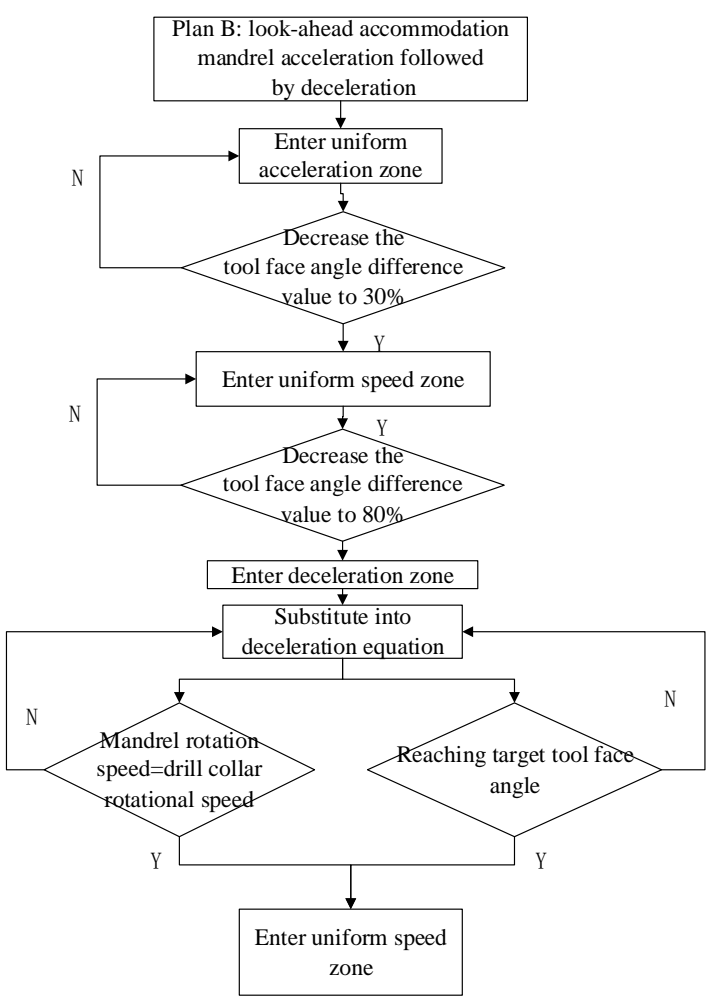

(b) Plan B

Figure 3: Control algorithm of mandrel look-ahead or lag speed control 
(1) Uniform deceleration section (initial section): mandrel rotational speed is changed according to the following formula 2:

$$
n(i)=n(i-1)-a
$$

$\Delta n(i)$ is always a constant(-a) at the stage, and mandrel accepts uniform deceleration motion. Uniform deceleration zone starts from the initial stage to $\left|\beta-\beta_{0}\right| \star 30 \%$.

(2) Constant speed section (buffer section): mandrel rotational speed is changed according to the following formula 3:

$$
n(i)=n(i-1)
$$

Mandrel rotational speed is always constant at the stage, and the mandrel maintains motion of uniform speed. Uniform speed zone starts from the initial stage to $\left|\beta-\beta_{0}\right| \star 80 \%$ (the parameter can be adjusted according to actual condition). ( $\beta$ Stands for target tool face angle, and $\beta_{0}$ stands for initial tool face angle).

(3) Variation acceleration section (final section): mandrel rotational speed is changed according to the following formula 4:

$$
\begin{aligned}
n(i) & =n(i-1)+K N^{\star}\left(n_{\text {drill collar }}-n(i-1)\right) \\
& +K T^{\star}\left(T F_{\text {target }}-T F_{\text {current }}\right)
\end{aligned}
$$

$K N$ - Mandrel rotational speed difference control coefficient, KT - Tool surface difference control conefficient.

$T F_{\text {target }}$ stands for target tool face, and $T F_{\text {current }}$ stands for current tool face.

The increment of mandrel rotational speed at each moment of the stage is changed constantly as it approaches the target parameter. The mandrel undergoes variation acceleration motion. Acceleration is larger and the change is faster at the initial stage. When the target parameter is approached, the acceleration is decreased. When mandrel rotational speed reaches drill collar rotational speed, and tool face angle reaches the target tool face angle $\beta$, mandrel rotational speed begins to align with drill collar rotational speed.

\section{Simulation result}

Initial setting:

- Mandrel rotational speed $=$ drill collar rotational speed $=400 \mathrm{rpm}$;
- Maximum acceleration (deceleration) speed: $16.67 \mathrm{rps}^{2}$;

- Uniform deceleration (acceleration) zone: $\left|\beta-\beta_{0}\right|$ * $30 \%$;

- Constant speed zone: $\left|\beta-\beta_{0}\right|$ * $80 \%$;

- Control rate (feedback rate): $2 \mathrm{~ms}$.

Since different target tool face angles and initial tool face angles are selected, 4 circumstances in section 2.1 appear, and 4 circumstances are respectively discussed accordingly:

(1) $\left|\beta-\beta_{0}\right| \leq 180^{\circ}$ and $\beta-\beta_{0}>0^{\circ}$ :

If initial tool face angle is set to be $\beta_{0}=60^{\circ}$, and target tool face angle is set to be $\beta=-60^{\circ}$ :
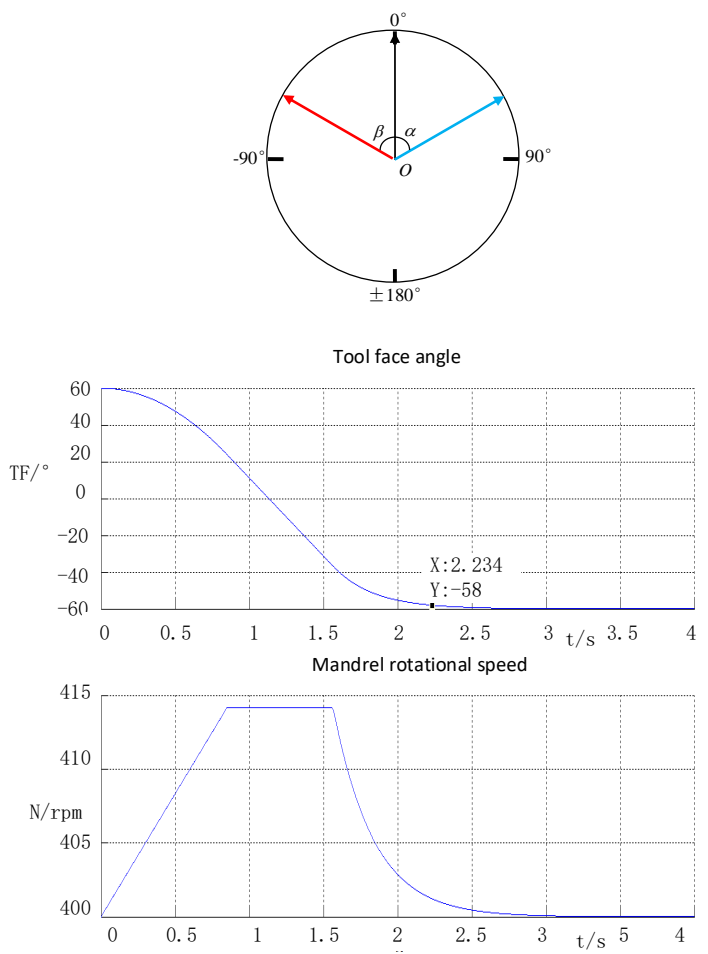

Figure 4: $\mid$ difference value $\mid \leq 180^{\circ}$ and difference value $>0^{\circ}$

Figure 4 shows that when the tool face angle is changed from $60^{\circ}$ to $-60^{\circ}$, mandrel should first be accelerated followed by deceleration, and when the tool face angle reaches $-58^{\circ}$, mandrel rotational speed is $401.2 \mathrm{rpm}$, and time is $2.234 \mathrm{~s}$.

(2) $\left|\beta-\beta_{0}\right| \leq 180^{\circ}$ and $\beta-\beta_{0}<0^{\circ}$ :

If initial tool face angle is set to be $\beta_{0}=-60^{\circ}$, and target tool face angle is set to be $\beta=60^{\circ}$ : 

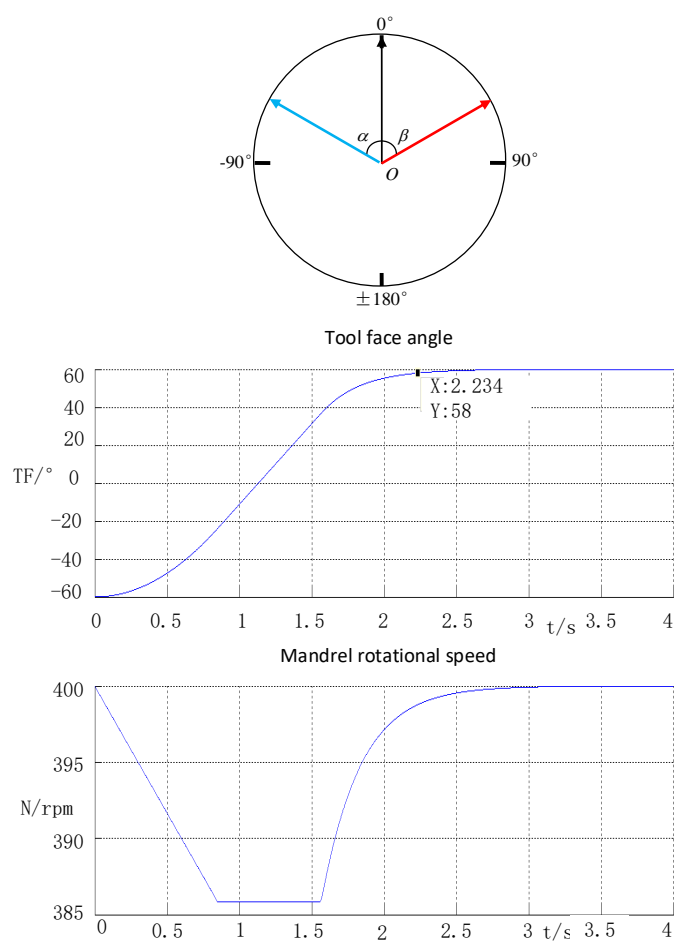

Figure 5: $\mid$ difference value $\mid \leq 180^{\circ}$ and difference value $<0^{\circ}$

Figure 5 shows that when the tool face angle is changed from $-60^{\circ}$ to $60^{\circ}$, mandrel should first be decelerated followed by acceleration, and when tool face angle reaches $58^{\circ}$, mandrel rotational speed is $398.8 \mathrm{rpm}$, and time is $2.234 \mathrm{~s}$.

(3) $\left|\beta-\beta_{0}\right| \geq 180^{\circ}$ and $\beta-\beta_{0}>0^{\circ}$ :

If initial tool face angle is set to be $\beta_{0}=60^{\circ}$, and target tool face angle is set to be $\beta=-150^{\circ}\left(210^{\circ}\right)$ :

Figure 6 shows that when tool face angle is changed from $60^{\circ}$ to $-150^{\circ}\left(210^{\circ}\right)$, mandrel should first be decelerated followed by acceleration, and when tool face angle reaches $208^{\circ}$, mandrel rotational speed is $398.9 \mathrm{rpm}$, and time is $2.568 \mathrm{~s}$.

(4) $\left|\beta-\beta_{0}\right| \geq 180^{\circ}$ and $\beta-\beta_{0}<0^{\circ}$ :

If initial tool face angle is set to be $\beta_{0}=-150^{\circ}$, and target tool face angle is set to be $\beta=60^{\circ}\left(-300^{\circ}\right)$ :

Figure 7 shows that when the tool face angle is changed from $-150^{\circ}$ to $60^{\circ}\left(-300^{\circ}\right)$, the mandrel should first be accelerated followed by deceleration, and when the tool face angle reaches $-298^{\circ}$, mandrel rotational speed is $401.1 \mathrm{rpm}$, and time is $2.568 \mathrm{~s}$.

In summary, the above four conditions are simulated, and it is known that the formulated control strategy can real-

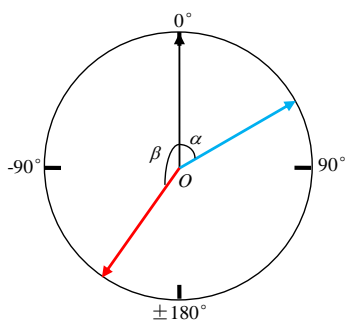

Tool face abgle
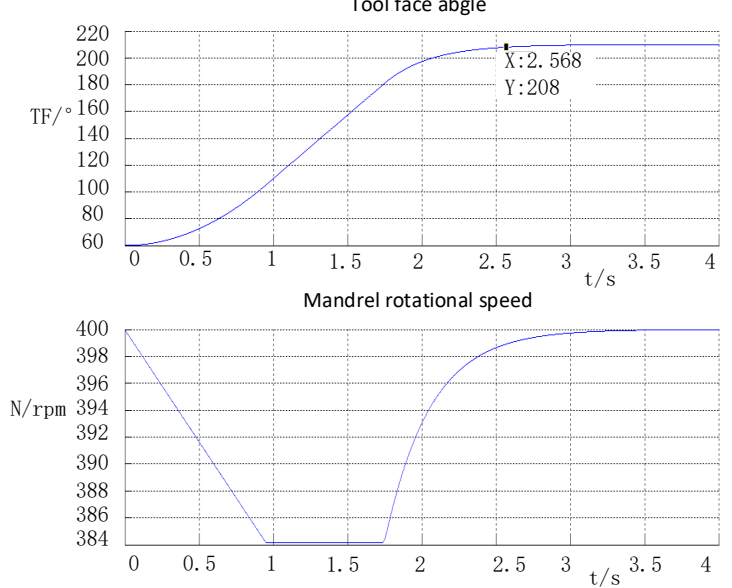

Figure 6: $\mid$ difference value $\mid \geq 180^{\circ}$ and difference value $>0^{\circ}$

ize tool face angle change along the shortest path. Control mandrel rotational speed can reach drill collar rotational speed $[13,14]$. Meanwhile, the tool face also can reach the anticipated control position. In other words, the double control of the mandrel nature speed closed loop and tool face angle position closed loop can be realized at the same time. The simulation result proves the feasibility and correctness of the formulated control strategy and algorithm.

\section{Key parameter selection method}

The control equation in formula 4 as follows in the drill collar acceleration (deceleration) motion through kinematics analysis:

In formula 4, $K N$ and $K T$ are two control coefficients, and they are the two most critical parameters in the whole control flow, which determines the rapidity and stability of the whole set control system. Selection method of the two parameters is mainly analyzed as follows [15]:

$$
\begin{aligned}
n(i) & =n(i-1)+K N^{\star}\left(n_{\text {drill collar }}-n(i-1)\right) \\
& +K T^{\star}\left(T F_{\text {target }}-T F_{\text {current }}\right)
\end{aligned}
$$



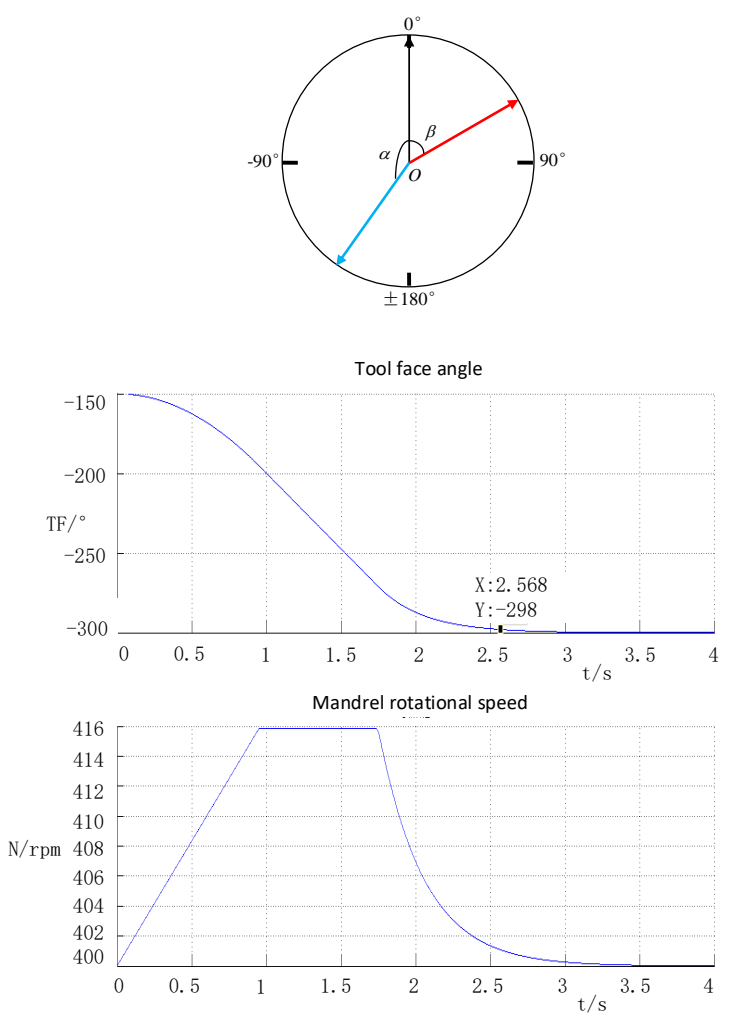

Figure 7: $\mid$ difference value $\mid \geq 180^{\circ}$ and difference value $<0^{\circ}$

(1) $K N$ : it is determined by drill collar rotation acceleration. Constant can be set for $K N$ accordingly, $K N=$ $16.67 \mathrm{rps}^{2}$ in simulation result, and it is equal to acceleration (deceleration) speed of uniform acceleration (deceleration) motion.

(2) $K T$ : It is determined by difference value between target tool face angle and initial tool face angle, if $K T$ is also set as constant:

When $K T=0.5$, the initial tool face angle $=0^{\circ}$, and when the target tool face angles are $30^{\circ}, 60^{\circ}$ and $120^{\circ}$ respectively, the simulation results of the mandrel rotational speeds variation are shown as Figures 8(a), 8(b) and 8(c).

The position marked by the red circle in Figure 8 shows the change of rotational speed transition stage of constant speed zone and variation acceleration zone. The acceleration in Figure 8(a) is increased quickly; $a=206.94 \mathrm{rps}^{2}$. This acceleration is higher than the maximum acceleration of the actual mandrel motor. The acceleration in Figure 8 (b) is increased more gently, acceleration $a=45.4 \mathrm{rps}^{2}$, which is close to maximum acceleration of actual mandrel motor. Figure 8(c) shows vibration, which leads to poor system stability.

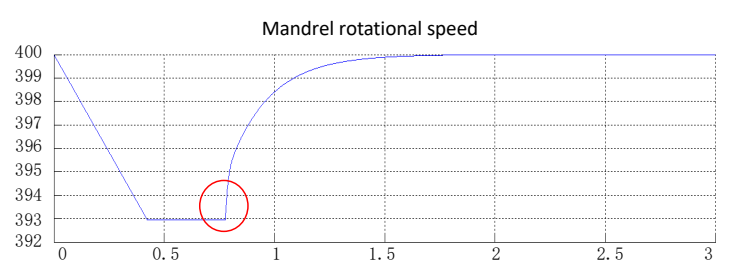

(a) difference value $=30^{\circ}$

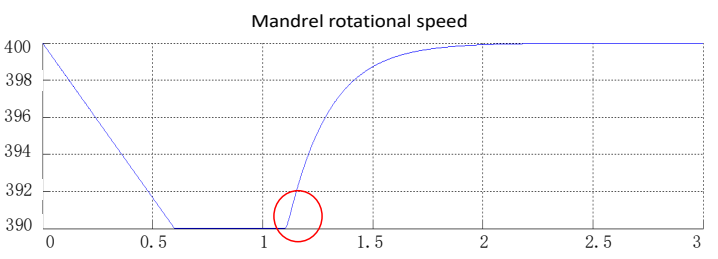

(b) difference value $=60^{\circ}$

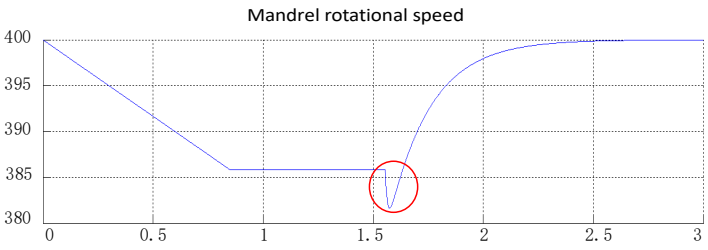

(c) difference value $=120^{\circ}$

Figure 8: Simulation results of Mandrel rotational speeds

It is obvious that the difference values of target tool face angle and initial tool face angle are different. $K T$ is not constant. The parameter $K T$ needs to change with the difference value of target tool face angle and initial tool face angle in order to guarantee system stability and rapidity so as to realize the adaptive change and achieve the optimal control. Table 1 shows the optimum value of $K T$ under difference value conditions of different target tool face angles and initial tool face angles.

Data in Table 1 undergo curve fitting for obtaining the functional relationship between $K T$ and the difference value Theta_delta of target tool face angle and initial tool face angle.

Curve fitting results are shown as follows:

$$
K T=3.8451 \text { * }(\text { Theta_delta })^{-0.499}
$$

The autocorrelation coefficient $R^{2}=0.9996 \approx 1$, which proves that equation 5 has good fitting result, which is close to the ideal curve. 
Table 1: the optimum value of KT

\begin{tabular}{c|c|c|c|c|c|c|c}
\hline Theta & 5 & 10 & 15 & 20 & 30 & 40 & 50 \\
KT & 1.75 & 1.2 & 1 & 0.85 & 0.7 & 0.6 & 0.55 \\
\hline Theta & 60 & 70 & 80 & 90 & 100 & 110 & 120 \\
KT & 0.5 & 0.46 & 0.43 & 0.41 & 0.39 & 0.37 & 0.35 \\
\hline Theta & 130 & 140 & 150 & 160 & 170 & 180 & \\
KT & 0.34 & 0.33 & 0.31 & 0.3 & 0.295 & 0.29 & \\
\hline
\end{tabular}

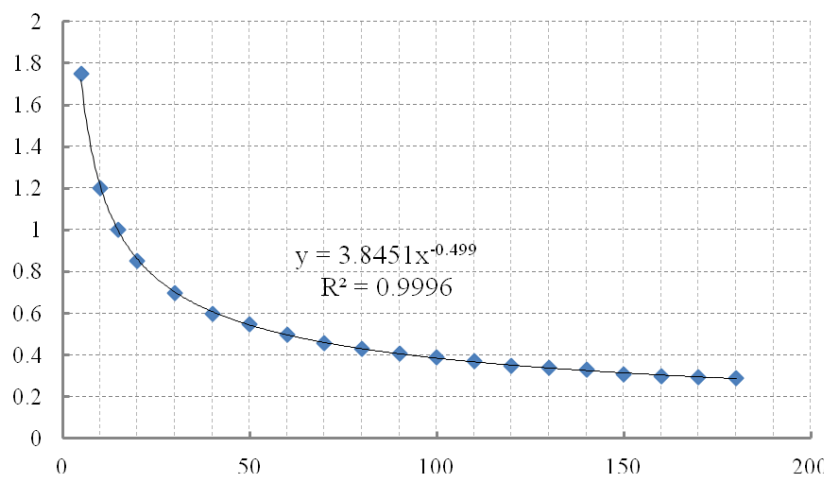

Figure 9: Curve fitting results

Therefore, the parameter $K T$ obtained from formula 5 is substituted into formula 4 for realizing the optimal control of the acceleration (deceleration) section.

\section{Conclusion}

Double closed-loop control of rotational speed loop and tool face angle position loop is realized through controlling mandrel rotational speed, relative rotational speed as zero of drill bit shaft (or mandrel) and drill collar is realized within the shortest time. The rotational speed accommodation decision control flow under mandrel lookahead or lag circumstance is proposed. Mandrel lag condition is adopted as an example for analyzing the control flow and algorithm. The two most critical parameters, $K N$ and $K T$, in the control flow are discussed, and the reliability of parameter selection is verified by autocorrelation algorithm and curve fitting. The simulation result shows that the method can better realize mandrel rotational speed adjustment and tool face change at any position aloge a $360^{\circ}$ circumference regardless of relative position of the mandrel and the drill collar.The mandrel can quickly track the rotational speed change of the outer drill collar, thereby realizing quick, stable and accurate steering functions. This paper has significance as a reference for the prototype production of a directional rotary steering wheel system.
Acknowledgement: This work was co-supported by the National Key R\&D Program of China (2016YFC0600301 and 2017YFC0602204-01).

\section{References}

[1] Zhang C., Peng L., Ma Y., Translated from Directional rotary steering drilling system angle position measurement method, Oil Drilling Techn., 2015, 37, 1-4.

[2] Teng X., Li K., Zeng Q., Translated from Research on control method of four-wheel drive omni-directional chassis, Comp. Measurem. Contr., 2015, 23, 1549-1553.

[3] Su Y., Translated from Research progress of downhole control engineering, Petrol. Industr. Publ. House., 2001, 20-21.

[4] Hu J., Zhou J., Fu X., Translated from Realization of closed-loop control of borehole trajectorywith controllable eccentricity, Nat. Gas Industry., 2002, 22, 59-61.

[5] Zhang G., Zhan M., Translated from Design of novel full rotary dynamic directional rotary steering drilling system, Petrol. Machin., 2016, 13-17.

[6] Su Y., Dou X., Wang J., Translated from Rotary steering drilling system function, characteristic and typical structure, Oil Drilling Techn., 2003, 25, 5-7.

[7] Brown T., Du. S, Eruslu S., Sayas F., Analysis of models for viscoelastic wave propagation, Appl. Math. Nonlin. Sci., 2018, 3, 55-69.

[8] Wang W., Wang Y., Translated from Rotational speed tracking control of permanent magnet synchronous motor, Systems Sci. Math., 2015, 9, 1028-1036.

[9] Li P., Jin F., Translated from Rotational speed tracking control of permanent-magnet synchronous motor, J. Shanghai Motor Institute, 2011, 6, 386-390.

[10] Alhihi M., Khosravi M. R., Formulizing the Fuzzy Rule for TakagiSugeno Model in Network Traffic Control, The Open Electr. Electr. Eng. J., 2018, 12, 1-11.

[11] Park J., Kim J., Park B., Myung H., Design and analysis of a new hybrid rotary steerable system for directional drilling, Int. Symp. Robotics., 2014, 1-3.

[12] Dewasurendra M., Vajravelu K., On the Method of Inverse Mapping for Solutions of Coupled Systems of Nonlinear Differential Equations Arising in Nanofluid Flow, Heat and Mass Transfer, Appl. Math. Nonlin. Sci., 2018, 3, 1-14.

[13] Ekstrand B. Continuous-Time Tracking Filters for the Coordinated Turn Model, The Open Aerospace Eng. J., 2009, 2, 6-19.

[14] Ousaloo H. S., Hysteresis Nutation Damper for Spin Satellite, The Open Aerospace Eng. J., 2013, 6, 1-5.

[15] Ballantyne R., Oppelt J., A Hybrid Approach to Closed-loop Directional Drilling Control using Rotary Steerable Systems, IFAC Proc. Vol., 2012, 45,84-89. 